\title{
Salud Percibida por los Pacientes en DPCA y DPA
}

Macarena Reina Neyra - Francisco Cirera Segura - Jesús Lucas Martín Espejo

Servicio de Nefrología - H.H.U.U. Virgen del Rocío. Sevilla

\section{RESUMEN}

La calidad de vida relacionada con la salud es un concepto multidimensional que permite identificar las dimensiones de la salud afectadas por la enfermedad renal crónica y podría ser una herramienta útil para evaluar el impacto de la diálisis peritoneal.

Se valora la utilidad del cuestionario SF-36v2 en pacientes sometidos a diálisis peritoneal, y cómo influyen variables demográficas y la modalidad de diálisis peritoneal sobre la salud percibida por estos pacientes.

Estudio multicéntrico descriptivo transversal, con una muestra de 89 pacientes en diálisis peritoneal. La edad media fue de 60,31 años. Para valorar la salud percibida utilizamos el SF-36v2 comparamos puntuaciones según el género, edad y modalidad de tratamiento.

Las mujeres mostraron puntuaciones menores; el grupo de 25-34 años tuvo puntuaciones más altas que los pacientes mayores, aunque estos últimos estaban más cerca de la población general. No aparecen diferencias significativas entre modalidades de tratamiento, incluso cuando se obviaron los factores que más influyen en la calidad de vida relacionada con la salud.

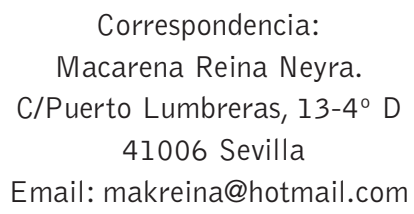

Correspondencia:

Macarena Reina Neyra.

C/Puerto Lumbreras, $13-4^{\circ} \mathrm{D}$

41006 Sevilla

Email: makreina@hotmail.com

\section{PALABRAS CLAVE: \\ - DIÁLISIS PERITONEAL \\ - SF-36 \\ - CALIDAD DE VIDA \\ - ENFERMERÍA}

\section{Health Perceived by Patients in CAPD and APD}

\section{Abstract}

The quality of life related to health is a multidimensional concept that identifies the dimensions of health affected by chronic kidney disease and could be a useful tool to assess the impact of peritoneal dialysis.

The utility of the SF-366v2 questionnaire in patients undergoing peritoneal dialysis is evaluated, and how demographic variables and the type of peritoneal dialysis affect the health perceived by these patients.

Transversal descriptive, multicentre study, with a sample of 89 patients on peritoneal dialysis. The average age was 60.31 years. In order to evaluate the perceived health, we used SF-36v2 and compared scores according to gender, age and type of treatment.

Women reflected lower scores: the 25-34 year-old age group had higher scores than older patients, although the scores of the latter were closer to the general population. No significant differences were found between types of treatment, even when the factors with the greatest influence on quality of life related to health were disregarded. 


\section{PALABRAS CLAVE: \\ PERITONEAL DIALYSIS \\ SF-36 \\ QUALITY OF LIFE \\ NURSING}

\section{Introducción}

En nuestro país los pacientes en diálisis peritoneal (DP) se benefician de numerosos avances técnicos gracias a los cuales han disminuido el número de peritonitis, los líquidos peritoneales son más fisiológicos y pretenden evitar el deterioro del peritoneo a medio y largo plazo, en conjunto se ha mejorado la calidad y la dosis de tratamiento que reciben los pacientes de DP.

No siempre estos avances e incluso la elección de la técnica de diálisis se han relacionado con la salud percibida por los pacientes en términos de calidad de vida relacionada con la salud (CVRS), existiendo pocos estudios que hayan investigado esta relación ${ }^{1-4}$.

El término CVRS ha sido muy utilizado en las distintas áreas de la ciencia, siendo cada vez mayor la importancia de su medida para completar los resultados clínicos y bioquímicos obtenidos con el tratamiento con DP, e integrarlo en el modelo biológico de salud ${ }^{5}$. Se entiende por CRVS la evaluación subjetiva de la influencia del estado de salud, los cuidados sanitarios y la promoción de la salud, sobre la capacidad del individuo para tener un nivel de funcionamiento que le permita seguir aquellas actividades que le son importantes y afectan a su bienestar, dentro del concepto de calidad de vida tal y como lo define la $0 M^{6}$. Al ser la CVRS un concepto multidimensional basado en la percepción subjetiva del paciente, se puede explicar aquella sintomatología que a veces encontramos en los pacientes en DP, no relacionadas con los resultados bioquímicos obtenidos con el tratamiento, además existe una estrecha relación entre el estado de salud percibida y la mortalidad $^{7-8}$

Para medir la CVRS existen diferentes instrumento genéricos, uno de los más extendidos y utilizados por la comunidad científica es el SF-369, que permite valorar al paciente en tratamiento con DP, facilitando la identificación de aquellas dimensiones de la sa- lud que están afectadas por la enfermedad, y orientando el diseño de las actividades de Enfermería más apropiadas para cada paciente. Este cuestionario podría ser una herramienta necesaria para poder evaluar el impacto del tratamiento con DP, si consideramos que la CVRS es un indicador de la calidad de los cuidados y de la valoración de resultados en los diferentes tratamientos ${ }^{5-10}$ (DPCA o DPA) con DP.

\section{Objetivos}

El presente trabajo trata de abordar los siguientes objetivos:

- Valorar la utilidad del cuestionario SF-36v2 para Enfermería cuando es aplicado a una muestra de pacientes en DP.

- Valorar la influencia de los datos demográficos sobre la CVRS en nuestra muestra y compararlos con los valores correspondientes dados para la población general española.

- Valorar si existen diferencias significativas en la CVRS según la modalidad de DP utilizada.

\section{Material y Métodos}

Se diseñó un estudio descriptivo transversal o de corte, con una duración de 6 meses, cuya muestra estaba constituida por un total de 89 pacientes: 49 mujeres y 40 hombres, con enfermedad renal crónica (ERC) en tratamiento con DP en sus distintas modalidades, que pertenecían a 4 centros de nuestra comunidad autónoma (Virgen del Rocío y Virgen Macarena, de Sevilla; Carlos Haya, de Málaga y Puerto Real, de Cádiz).

Los criterios de inclusión fueron: a) Participación voluntaria en el estudio. b) Haber permanecido durante los últimos tres meses en DP. c) Ausencia de patología psiquiatría o deficiencia mental.

Para valorar la CVRS en el presente estudio, se utilizó el cuestionario de salud SF-36v2 cuya adaptación ha sido realizada para España por Alonso y cols. ${ }^{11-12}$, de SF-36 Health Survey ${ }^{13-14}$.

Este cuestionario consta de 36 ítems de carácter genérico que informan tanto de estados positivos como negativos de la salud física y del bienestar emocio- 
nal, identificando 8 dimensiones de salud: función física (FF), limitaciones de rol por problemas físicos (RF), dolor corporal (D), salud general (SG), vitalidad (V), función social (FS), limitaciones de rol por problemas emocionales (RE) y salud mental (SM). Se incluye un ítem que evalúa el cambio en la percepción del estado de salud actual respecto al año anterior (CS). En cada ítem la puntuación obtenida es transformada en una escala de 0 a 100 según el porcentaje alcanzado de la máxima puntuación posible, donde los valores más altos, indican una mejor calidad de vida.

Se facilitó el cuestionario a los pacientes para su autocumplimentación y en los casos de pacientes con problemas de lectura o compresión, deterioro auditivo o visual, se les realizó el cuestionario mediante un enfermero/entrevistador en cada centro. Junto con el cuestionario se recogieron datos demográficos (edad y sexo del paciente), la modalidad de DP utilizada (DPCA o DPA), el tiempo de permanencia en la misma y la hemoglobina.

Para comparar la muestra con las normas poblacionales en España y siguiendo las indicaciones del grupo español de validación del SF-3615, se estandarizaron las puntuaciones. El resultado viene dado en desviaciones estándar respecto a la población general de igual edad y sexo.

El análisis de los datos se realizó con el paquete estadístico SPSS. 14. Se utilizó para la descripción de las diferentes variables la media y la desviación es- tándar (DE). Los datos cualitativos se expresaron mediante frecuencias y porcentajes. Para el contraste entre variables se utilizó la T de Student o la prueba de Kruskall-Wallis según correspondiera. Para el estudio de correlaciones, se utilizó la Rho de Spearman. Se consideraron significativos los contrastes cuando la significación fue inferior al 5\%.

\section{Resultados}

La muestra la formaron 89 pacientes con ERC en DP. La edad media de los individuos fue de 60,31 + 15,89 años, con un ligero predomino del género femenino $(55,1 \%$,$) . No existió diferencia entre los ni-$ veles de hemoglobina en ninguno de los grupos, que se encontraban entre $122+12 \mathrm{~g} / \mathrm{L}$. Los pacientes se distribuían de forma similar entre DPCA $(n=45)$ y DPA $(n=44)$. La media del tiempo en DP fue de 22,35 (3-117) meses, sin existir diferencias significativas entre las dos modalidades.

A continuación se estudiaron las propiedades psicométricas de las diferentes escalas. Las pruebas estadísticas que determinan la consistencia interna del cuestionario presentan una fiabilidad alta, ya que todos los valores del test alfa de Cronbach se sitúan por encima del dintel de la normalidad situado en 0,7 , concretamente entre 0,738 (SG) y 0,951 (RE), hallándose 3 dimensiones con coeficientes superiores al valor de 0,9 . Las correlaciones entre las distintas dimensiones fueron todas significativas $(p<0,001)$ resultaron la $r^{2}$ en rangos intermedios. Las puntuaciones medias de cada una de las dimensiones del cuestionario, variaron entre 74,43 $\pm 29,67$ (RE) y $38,94 \pm 17,93$ (SG).

\begin{tabular}{|c|c|c|c|c|c|c|c|c|}
\hline & \multicolumn{4}{|c|}{ HOMBRES $(n=40)$} & \multirow{2}{*}{$\mathbf{p}^{1}$} & \multicolumn{3}{|c|}{ MUJERES $(n=49)$} \\
\hline & Media & & DE & Rango & & Media & DE & Rango \\
\hline $\mathbf{F F}$ & 70,12 & $\uparrow$ & 27,23 & $0-100$ & 0,039 & $56,73 \downarrow$ & 32,05 & $0-100$ \\
\hline RF & 60,68 & $\uparrow$ & 29,63 & $0-100$ & Ns & $56,12 \downarrow$ & 33,47 & $0-100$ \\
\hline D & 66,27 & $\uparrow$ & 28,50 & $0-100$ & Ns & $56,65 \downarrow$ & 30,14 & $0-100$ \\
\hline SG & 40,37 & $\uparrow$ & 16,44 & $5-77$ & Ns & $37,77 \vee$ & 19,15 & $0-77$ \\
\hline V & 52,18 & $\uparrow$ & 24,41 & $0-93$ & Ns & $43,62 \vee$ & 27,76 & $0-93,5$ \\
\hline FS & 71,25 & $\uparrow$ & 28,19 & $12,50-100$ & Ns & $65,30 \vee$ & 35,47 & $0-100$ \\
\hline RE & 81,45 & $\uparrow$ & 24,92 & $16,67-100$ & 0,043 & $68,70 \vee$ & 32,16 & $0-100$ \\
\hline SM & 68,12 & $\uparrow$ & 20,11 & $5-90$ & Ns & $59,79 \vee$ & 23,13 & $10-100$ \\
\hline
\end{tabular}

Se analizó la CVRS según el género de los pacientes (tabla 1), siendo superior la puntuación en el género masculino en todos los casos, obteniendo diferencias significativas en la FF $(p=0,039)$, y el RE $(p=0,043)$. Según la edad (tabla 2), se encontraron diferencias significativas en todas las dimensiones excepto para la SG, RE y SM, obteniendo puntuaciones más altas que el grupo de menor edad.

TABLA 1. Distribución de las puntuaciones del SF-36, según el género. 


\begin{tabular}{|c|c|c|c|c|c|c|}
\hline \multirow{2}{*}{ Edad } & $25-34(n=8)$ & $35-44(n=12)$ & $45-54(n=9)$ & $55-64(n=17)$ & $65-74(n=22)$ & $>75(n=21)$ \\
\hline & Media (DE) & Media (DE) & Media (DE) & Media (DE) & Media (DE) & Media (DE) \\
\hline FF1 & $89,37^{\uparrow}(11,47)$ & $75(19,88)$ & $71,11(23,28)$ & $75,29(22,87)$ & $9,54(28,49)$ & $35,23 \downarrow(31,83)$ \\
\hline $\mathbf{R F}^{3}$ & $77,35 \uparrow(23,83)$ & $52,61(27,49)$ & $61,11(27,38)$ & $71,07(30,65)$ & $58,24(33,09)$ & $42,26 \downarrow(32,10)$ \\
\hline D2 & $81,87^{\uparrow}(16,66)$ & $72,25(24,58)$ & $70,11(29,81)$ & $66,70(29,14)$ & $56,77(29,07)$ & $42,42 \downarrow(28,58)$ \\
\hline SG & $50,25^{\uparrow}(23,31)$ & $40,41(13,92)$ & $42,22(11,15)$ & $41,41(13,04)$ & $39,22(21,21)$ & $30,09 \downarrow(17,75)$ \\
\hline v2 & $67,18^{\uparrow}(21,32)$ & $51,56(25,71)$ & $55,55(19,62)$ & $57,72(24,35)$ & $46,59(23,04)$ & $26,78 \downarrow(25,47)$ \\
\hline FS $^{2}$ & $75(25,87)$ & $61,45(27,41)$ & $79,16(25,76)$ & $82,35 \uparrow(27,97)$ & $75(31,57)$ & $45,23^{\downarrow}(33,88)$ \\
\hline RE & $85,41^{\uparrow}(22,15)$ & $76,38(21,56)$ & $69,44(28,56)$ & $81,86(26,39)$ & $77,65(28,21)$ & $61,90^{\downarrow}(37,96)$ \\
\hline SM & $71,87^{\uparrow}(15,56)$ & $57,91^{\downarrow}(23,10)$ & $66,66(13,91)$ & $70,58(18,61)$ & $62,04(27,06)$ & $58,09(22,82)$ \\
\hline \multicolumn{7}{|c|}{${ }^{1}$ Prueba Kruskall-Wallis con $\mathbf{p}<0,001$ 2Prueba Kruskall-Wallis con $\mathbf{p}<0,01.3$ Prueba Kruskall-Wallis con $\mathrm{p}=0,05$} \\
\hline \multicolumn{7}{|c|}{$\begin{array}{l}\text { FF: función física; RF: limitaciones de rol por problemas físicos D: dolor corporal, SG: salud general; V: vitalidad; FS: función social; RE: limitacio- } \\
\text { nes de rol por problemas emocionales; SM: salud mental.. }\end{array}$} \\
\hline
\end{tabular}

TABLA 2. Distribución de las puntuaciones del SF-36, según la edad.

Las puntuaciones de la muestra se estandarizaron para compararlas con las descritas para la población general española y se muestran en la figura 3, donde todas las dimensiones fueron significativas $(p<0,001)$, excepto RF $(p=0,013), \operatorname{RE}(p=0,002)$ y SM $(p=0,004)$, y por grupos de edad (figura 4$)$, donde fue significativa la puntuación de la SG $(p=0,023)$ en el grupo de 25 a 34 años, la FF $(p=0,017), S G \quad(p<0,001), V \quad(p=0,020), F S$ $(p=0,06)$ y $S M(p=0,014)$ en el grupo de 35 a 44 . EI RF $(p=0,041)$ y $S G(p<0,001)$ fueron significativos en el grupo de 45 a 54, la SG fue significativa en los grupos de 55 a 64 y en el grupo de 65 a 74, con $p<0,001$ y $p=0,014$ respectivamente. En el gru-

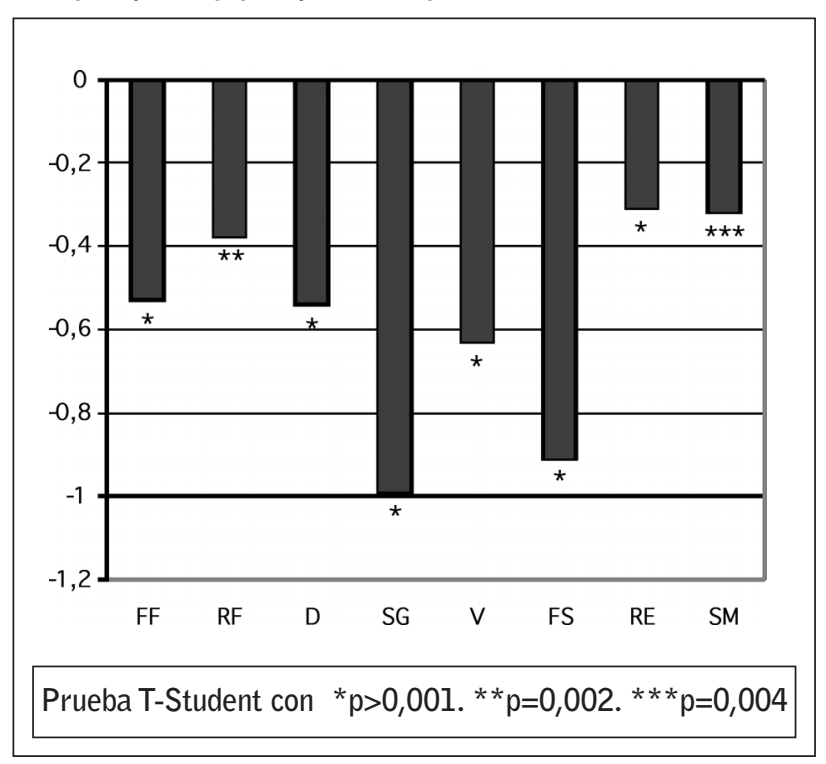

Figura 3. Comparación de las puntuaciones estandarizadas del SF-36. po de pacientes mayores de 75 años, fueron significativas todas las dimensiones $D, S G, V$ y $F S$ $(p<0,001)$ FF y RF $(p<0,01)$ y RE y $S M$ con $(p<0,05)$.

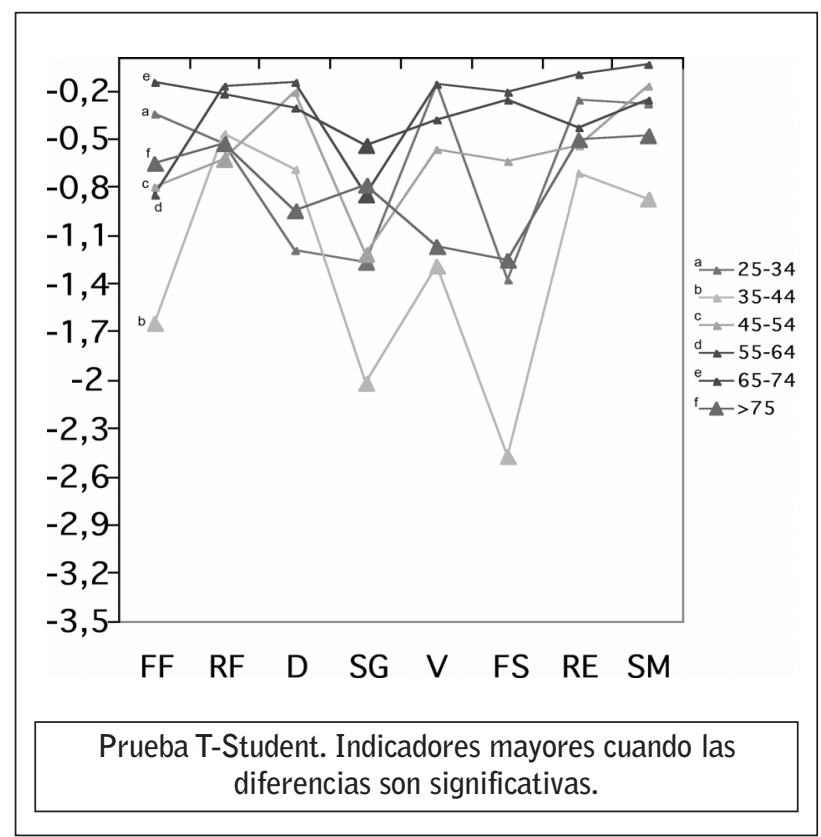

Figura 4. Comparación de las puntuaciones estandarizadas del SF-36, según la edad.

Se compararon las puntuaciones obtenidas en las 8 dimensiones del SF-36, en función de la modalidad de DP utilizada. Las puntuaciones más altas se dieron siempre en el grupo de DPA llegando a ser significativas en los casos de la FF $(p=0,03)$, SG $(p=0,017)$ y $\vee(p=0,016)$ (tabla 3$)$. 


\begin{tabular}{|c|c|c|c|c|c|c|c|}
\hline & \multicolumn{3}{|c|}{ DPCA $(n=45)$} & \multirow{2}{*}{$\mathbf{p}^{1}$} & \multicolumn{3}{|c|}{ DPA $(n=44)$} \\
\hline & Media & DE & Rango & & Media & DE & Rango \\
\hline $\mathbf{F F}$ & 53,44 & 34,12 & $0-100$ & 0,03 & $72,27 \uparrow$ & 23,16 & $0-100$ \\
\hline RF & 55,46 & 32,83 & $0-100$ & Ns & $60,94 \uparrow$ & 30,65 & $0-100$ \\
\hline D & 58,68 & 31,64 & $0-100$ & Ns & $63,31 \uparrow$ & 27,61 & $12-100$ \\
\hline SG & 34,48 & 16,58 & $0-62$ & 0,017 & $43,50 \uparrow$ & 18,29 & $0-77$ \\
\hline V & 40,83 & 26,26 & $0-75$ & 0,016 & $54,26 \uparrow$ & 25,28 & $0-93$ \\
\hline FS & 64,16 & 34,91 & $0-100$ & Ns & $71,87 \uparrow$ & 29,42 & $0-100$ \\
\hline RE & 72,22 & 31,53 & $0-100$ & Ns & $76,70 \uparrow$ & 27,81 & $0-100$ \\
\hline SM & 63,4 & 22,90 & $10-100$ & Ns & $63,63 \uparrow$ & 21,52 & $5-100$ \\
\hline
\end{tabular}

Tabla 3. Puntuaciones del SF-36, según la modalidad de DP.

En la tabla 4 se observan las puntuaciones de ambas modalidades de tratamiento, discriminando los dos factores que más influyen en la calidad de vida, edad y sexo, determinando así la influencia de la técnica en la CVRS.

En los pacientes menores de 63 años, cuando eran hombres tanto la DPCA como la DPA se mostraron similares respecto a la CVRS, siendo la DPCA en el caso de las mujeres, la que obtuvo las puntuaciones más altas. Cuando los pacientes eran mayores de 63, años en ambos casos la DPA obtuvo las puntuaciones más altas llegando a ser significativas en el caso de las mujeres en las dimensiones de $S G(p=0,048)$ y $V$ $(p=0,033)$.



Tabla 4. Comparación de puntuaciones según la técnica, discriminando sexo y edad de los pacientes. 


\section{Discusión}

Como otros autores han descrito en sus trabajos, la CVRS es un importante indicador de la calidad de los cuidados y de la valoración de resultados en pacientes crónicos y pluripatológicos ${ }^{5-10}$. La valoración de la salud percibida por el paciente, implica ciertos problemas al ser un concepto subjetivo, universal, holístico y dinámico. Para este fin, se han elaborado instrumentos que permiten cuantificar en la medida de lo posible la CVRS. En este trabajo, hemos utilizado el cuestionario SF-36v2 en su versión española, mostrando el mismo características psicosométricas adecuadas y muy similares a las obtenidas en el estudio que determina los valores poblacionales de referencia en España ${ }^{15}$. Este cuestionario es fácil de administrar y rápido de cumplimentar.

Al aplicar el cuestionario a una muestra de pacientes con ERC en DP, obtuvimos unos índices de consistencia (alfa de Cronbach) superiores a 0,70, valor mínimo recomendado para hacer comparaciones de grupo y en tres de las dimensiones se superó el valor de 0,90 , que permite utilizar las puntuaciones del cuestionario a nivel individualizado. Las correlaciones internas de las 8 dimensiones tuvieron significación estadística en todos los casos, cumpliendo así los requisitos de validez del cuestionario, y demostrando que todas las dimensiones están interrelacionadas, de tal manera que cuando un individuo tiene deteriorada una de las funciones repercute sobre todas las demás. Por lo que consideramos este cuestionario una herramienta útil, que Enfermería puede utilizar para valorar las necesidades de los pacientes, en nuestro caso ERC en DP en cualquiera de sus modalidades de tratamiento. Además es válido a la hora de realizar una continuidad tanto del estado de salud como de los cuidados que requiere el paciente.

Los valores medios obtenidos para el conjunto de la muestra estudiada, se hallaron entre el 74,43 del RE y el 38,94 de la $S G$, estando todos los valores entorno a una puntuación media, demostrando que la ERC afecta a todas las dimensiones. En la muestra, las mujeres han obtenido puntuaciones más bajas en todas las dimensiones del cuestionario. Son numerosos los estudios que coinciden con nuestro resultado, tanto para los pacientes con ERC como en la población general ${ }^{15-17}$. Por lo que este hallazgo no se debe a la patología que nos ocupa. No existe una explicación clara de estas diferencias y tradicionalmente se atribuyen a los roles sociales de cada género. Este resultado se da también en otros indicadores utilizados para establecer la calidad de vida y rebate la suposición de que una mayor longevidad del género femenino, suponga una mayor calidad de vida. Incluso esta suposición puede llegar a invertirse cuando se tiene en cuenta la esperanza de vida libre de enferme$\mathrm{dad}^{15}$.

La edad es un factor que en nuestra muestra resultó significativo tanto para las dimensiones relacionadas con la salud física (FF, RF, D, V) como para el bienestar emocional ( $F S$ y $V$ ). Al igual que el género, la CVRS empeora con la edad, tanto en los pacientes con ERC como en la población general. Sin embargo, al estandarizar las puntuaciones usando normas poblacionales, comprobamos al igual que otros estudios10,16 que la CVRS en los grupos de mayor edad está más cercana a la población general, superando el estado de salud percibida a la de los grupos de menor edad. Así, en nuestra muestra el grupo de pacientes comprendido entre 65-74 años, tienen una mejor puntuación en todas las dimensiones que el grupo comprendido entre 18-24 años.

Este hecho puede deberse a que los pacientes de más edad ven menos afectada sus actividades diarias como pueden ser la vida laboral, familiar, social y sexual que los grupos de pacientes más jóvenes. No podemos olvidar que estamos utilizando un instrumento que determina la salud percibida por los pacientes. Siendo el grupo de pacientes en DP mayores de 75 años los que no se incluyen en esta afirmación, debido a las dificultades que los pacientes con estas edades suelen tener para el autocuidado y la realización de la técnica.

Cuando comparamos la DPCA y la DPA observamos que en todas las dimensiones las puntuaciones más altas se corresponden con la DPA, siendo sólo significativas la FF, la SG y la V, esto puede deberse a una mejoría de la función física y mental que facilita la vida social al tener más tiempo libre de tratamiento durante el día. A pesar de que en todas las dimensiones las puntuaciones en DPA son más altas, en este estudio no hemos encontrado diferencias significativas a la hora de valorar la CVRS según la modalidad de DP utilizada. Debemos tener en cuenta también, que no todos los pacientes son libres de cambiar de 
una técnica a otra o de incluso elegir la DPA como forma de tratamiento, debido a muchos motivos incluidos los económicos

Dos de los factores que hemos comprobado que influyen más en la CVRS son la edad y sexo de los pacientes. Por lo que agrupando a los pacientes por estas características y comparando las técnicas, observamos que no existen diferencias significativas entre las dos modalidades de DP, resultado que coincide con otros estudios publicados. ${ }^{2-4}$

\section{Conclusiones}

Consideramos que el cuestionario SF-36 es un instrumento válido y útil para valorar el estado de salud percibido por los pacientes con ERC en DP.

Hemos observado que la ERC y la DP afectan notablemente a la salud percibida de los pacientes respecto a la población general española de igual edad y sexo. El género femenino y la mayor edad del paciente se han mostrado indicadores de una peor CVRS dentro de la muestra.

Consideramos que sería necesario hacer estudios longitudinales para generalizar estos resultados, así como valorar la repercusión de las intervenciones de Enfermería sobre la calidad de vida.

\section{Agradecimientos}

Agradecemos las facilidades prestadas por todos los centros que han intervenido en el estudio, así como a las personas que han colaborado en la recogida de los datos.

\section{Bibliografía}

1. Ruiz Fernandez E. Evolución histórica de la diálisis peritoneal. En: Diálisis Peritoneal. Libro de comunicaciones del tercer curso andaluz de diálisis peritoneal para Enfermería. SEDEN. 2003.

2. Ardine de Wit G, P. Merckus M, T.Krediett R et al. A comparison of life of patients on automated and continuous ambulatory peritoneal dialysis. Peritoneal Dialysis International. 2001; 21:306-312.

3. Bro S, Bjorner J, Tofte-Jensen P, et al. A prospective, randomized multicenter study comparing APD and CAPD treatment. Peritoneal Dialysis International. 1999; 19: 526-533.

4. Rabindranath KS, Adams J, Ali TZ, et al. Diálisis peritoneal ambulatoria continua versus diálisis peritoneal automatizada para la nefropatía terminal (Revisión Cochrane traducida). En: La Biblioteca Cochrane Plus, 2007 Número 3. Oxford: Update Software Ltd. Disponible en: http://www.updatesoftware.com.

5. Valdés C, Ortega F. Avances en la calidad de vida relacionada con la salud y el trasplante renal. Nefrología. 2006. 26 (2).

6. Organización Mundial de la Salud. Quality of Life assessment. An annotated bibliography. Who (MNH/PSF/94.1). Géneve. 1994.

7. Kaplan GA, Camacho T. Perceived health and mortality: A nine year follow-up of the human population laboratoty cohort. Am Journal Epidemiol. 1983; 117:292-298.

8. McClellan WM, Anson C, Birkely K, et al. Functional status and quality of life: predictors of early mortality among patients entering treatment for end-stage renal disease. J Clin Epidemiol. 1991; 44: 83-89.

9. Iraurgi I, Póo M, Márkez I. Valoración del índice de salud SF-36 aplicado a usuarios de programa de metadona. Valores de referencia para la Comunidad Autónoma Vasca. Revista Española Salud Pública. 2004; 78: 609-621.

10. Gil JM, García MJ, Foronda J. Calidad de vida relacionada con la salud en pacientes ancianos en hemodiálisis. Nefrología. 2003; 23 (6).

11. Vilagut G, Ferrer M, Rajmil L. El cuestionario de salud SF-36 español: una década de experiencia y nuevos desarrollos. Gac Sanit. 2005; 19 (2):135-150. 
12. Alonso J, Prieto $L$, Antó JM. La versión española del SF-36 Health Survey (Cuestionario de Salud SF-36): un instrumento para la medida de los resultados clínicos. Med Clin 1995; 104: 771-776.

13. McHorney CA, Ware JE, Raczek AE. The MOS 36-item short form health survey (SF-36) II. Psychometric and clinical tests of validity in measuring psysical and mental health constructs. Med Care. 1993; 31:247-63.

14. McHorney CA, Ware JE, Lu R, Sherbourne CD. The MOS 36-item short form health survey (SF36) III. Tests of data quality scaling assumtions, and reliability across diverse patient groups. Med Care. 1994; 32:40-66.
15. Alonso J, Regidor E, Barrio G, et al. Valores poblacionales de referencia de la versión española del Cuestionario de Salud SF-36. Med Clin. 1988; 111: 410-416.

16. Vázquez I, Valderrábano F, Fort J, et al. Diferencias en la calidad de vida relacionada con la salud entre hombres y mujeres en tratamiento en hemodiálisis. Nefrología 2004; 24 (2). 